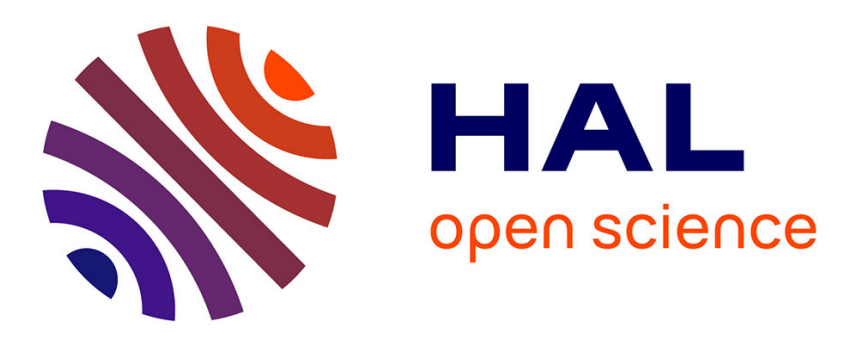

\title{
Variations observées dans l'état de développement des systèmes foliaire et racinaire chez la semence mûre de maïs
}

Marc Juguet, Maurice Derieux, Jean-Benoît Duburcq, Annie Diaz

\section{- To cite this version:}

Marc Juguet, Maurice Derieux, Jean-Benoît Duburcq, Annie Diaz. Variations observées dans l'état de développement des systèmes foliaire et racinaire chez la semence mûre de maïs. Agronomie, 1986, 6 (3), pp.299-304. hal-00884879

\section{HAL Id: hal-00884879 \\ https://hal.science/hal-00884879}

Submitted on 1 Jan 1986

HAL is a multi-disciplinary open access archive for the deposit and dissemination of scientific research documents, whether they are published or not. The documents may come from teaching and research institutions in France or abroad, or from public or private research centers.
L'archive ouverte pluridisciplinaire HAL, est destinée au dépôt et à la diffusion de documents scientifiques de niveau recherche, publiés ou non, émanant des établissements d'enseignement et de recherche français ou étrangers, des laboratoires publics ou privés. 


\title{
Variations observées dans l'état de développe- ment des systèmes foliaire et racinaire chez la semence mûre de maïs
}

\author{
Marc JUGUET, Maurice DERIEUX $\left({ }^{*}\right)$ \& Jean-Benoît DUBURCQ $\left({ }^{*}\right)$ \\ avec la collaboration technique de Annie DIAZ \\ Université de Picardie, Laboratoire de Botanique et d'Embryologie, 33 rue St Leu, F 80039 Amiens \\ (*) I.N.R.A., Laboratoire de Génétique et d'Amélioration des Plantes, Estrées-Mons, 80200 Péronne
}

Mots clés additionnels : Zea mays, embryon, feuilles, racines, variabilité génétique. in order to determine the capital of embryos available for germination. Among the differences observed was greater leaf development in hybrid A632 $\times$ W117.

Additional key words : Zea mays, embryo, leaves, roots, genetic variability.

\section{INTRODUCTION}

Le rayonnement intercepté par une culture est considéré comme étant l'un des principaux facteurs de la production (BISCOE \& GALLAGHER, 1977; MONTEITH, 1977 ; GOSSE et al., 1986). En particulier sur le maïs, plusieurs auteurs ont montré que la production totale de matière sèche pouvait être reliée au rayonnement intercepté (SEUNG KeUN JONG et al., 1982; BONHOMME et al., 1982).

Dans nos régions la culture du maîs s'implante très tard dans la saison. Le semis a lieu fin avril-début mai et la surface foliaire se met en place lentement. D'après VARLET-GRANCHER (1982), l'efficience d'interception $60 \mathrm{j}$ après le semis (1 ${ }^{\text {er }}$ juillet) n'est que de 15 p. 100 , alors que le rayonnement incident diminue dès la $2^{\mathrm{e}}$ moitié du mois de juillet. Un léger avantage durant cette période aurait donc des conséquences importantes. BLOC et al. (1979) ont signalé que le rythme d'apparition des feuilles ne variait pas sur une gamme d'une dizaine d'hybrides, mais que l'un des hybrides (A632 $\times$ W117) avait un avantage d'une feuille dès le stade 4-5 feuilles. Le nombre total de feuilles est étroitement lié à la précocité ; cet hybride semble garder un avantage d'environ 1 feuille par rapport à sa précocité.

Il nous a donc semblé important d'observer l'embryon pour essayer d'expliquer un avantage à un stade aussi jeune. L'objectif étant d'utiliser en sélection cette supériorité pour permettre une meilleure interception au cours du printemps et, peut être, la mise en place d'une surface foliaire plus grande à précocité égale. Nous présentons ici les premiers résultats de cette étude.

\section{II. ÉTUDE BIBLIOGRAPHIQUE}

Chez le maïs, l'embryon de la graine mûre (embryon adulte) a fait l'objet de 2 grands types d'études morphologiques: certains auteurs se sont intéressés à son poids, d'autres à l'état de développement atteint par ses différents organes. 
De nombreux travaux, essayant de relier le poids des embryons chez les lignées parentales et chez les hybrides ainsi que le poids de l'embryon à la vigueur de la plante, ou encore le poids de l'embryon à des caractères histologiques ou cytologiques, ont donné des résultats contradictoires (voir SHAFER, 1944 et FAIRCHILD, 1953).

Les recherches organogéniques sont moins nombreuses; nous citerons uniquement celles qui font mention de l'embryon adulte.

MARTIN \& HERSHEY (1935) signalent, sans illustration et sans mesure, 6 à 9 nœufs dans les variétés étudiées ; le $1^{\text {er }}$ nœud étant occupé par le coléoptile, il existerait donc 5 à 8 feuilles avant la germination.

RANDOLPH (1936) est le premier à étudier complètement l'embryogenèse ; pour lui, l'embryon adulte possède habituellement 5 feuilles et « a slight increase in size throughout the embryo ", phrase qui pourrait signifier l'existence d'un primordium de la $6^{\mathrm{e}}$ feuille. Cette affirmation n'est étayée par aucune illustration.

KIESSELBACH (1949) compte 3 ou 4 feuilles et 1 à 7 racines adventives selon les variétés.

SASS (1951) signale 4 ou 5 feuilles selon les hybrides, l'un d'entre eux possédant 5 feuilles et rarement 4.

HUBBARD (1951) (dans une thèse non publiée citée par BONNETT, 1953), étudiant 4 lignées trouve 4 ou 5 feuilles, la $5^{\mathrm{e}}$ étant parfois à l'état de primordium.

ABBE \& STEIN (1954) insistent sur la relativité de la notion d'embryon adulte (mature embryo), l'avancement maximal de l'organogenèse étant, pour une même lignée, fonction d'un grand nombre de facteurs : des conditions climatiques et de la constitution génétique évidemment, mais aussi de la date du semis et surtout de celle de la récolte. Dans la lignée observée, la majorité des embryons est au stade 5 (le plus jeune primordium reconnaissable est celui de la $5^{\text {e }}$ feuille) tandis qu'une petite fraction de la population a atteint le stade 6 .

BELL (1953) étudie 7 hybrides et indique que tous les embryons mûrs sont uniformes avec 5 feuilles.

SASS (1976), dans sa mise au point sur le développement du caryopse, conclut qu'il existe 5 feuilles sauf dans quelques lignées peu vigoureuses.

LOMASSON (1977) trouve également 5 feuilles.

Il semble évident, d'après les travaux que nous venons de citer, que la plupart des variétés possède 5 feuilles mais qu'il existe parfois des variations selon les lignées ou les hybrides ou exceptionnellement à l'intérieur d'une même variété. Cependant ces travaux sont peu précis car leurs auteurs ne définissent pas la notion de primordium ni surtout celle de feuille et ne pratiquent aucune mesure.

Seul FAIRCHILD (1953) a publié des résultats quantitatifs. Il compare le développement de 2 lignées de maïs significativement différentes par la taille de leurs organes à celui de leurs hybrides réciproques en mesurant la surface des organes sur des coupes transversales pratiquées à des niveaux déterminés ; la surface des feuilles, du coléoptile et du méristème caulinaire est mesurée au niveau d'initiation de la plus jeune feuille. Les 2 hybrides surpassent le parent le plus vigoureux, mais seul l'hybride « femelle vigoureuse $x$ mâle vigoureux » surpasse la lignée parentale vigoureuse $40 \mathrm{j}$ après la pollinisation. Par contre, aucun des 2 hybrides ne surpasse le parent le plus vigoureux en ce qui concerne la précocité d'initiation des racines séminales adventives.

Le travail de FAIRCHILD possède l'intérêt de supprimer les notions mal employées de primordium et de feuille ; la feuille est considérée en tant que telle dès le début de son initiation. Par contre, la mesure de la taille de la dernière feuille, toujours la $5^{\mathrm{e}}$, est sujette à caution ; en effet, comme nous l'avons constaté, la détermination de son niveau d'initiation est souvent difficile à apprécier sur les coupes transversales sériées. Ainsi avons-nous préféré mesurer sa longueur.

\section{MATÉRIEL, MÉTHODES ET TERMINOLOGIE}

Les caryopses sont réhydratés avec un mélange d'eau, de mouillant, d'acide acétique et de formol, de façon à empêcher toute germination; le non-réveil embryonnaire est vérifié par l'absence de mitoses. L'embryon extrait et le scutellum enlevé, les coupes transversales ou longitudinales sériées sont pratiquées uniquement sur l'axe embryonnaire.

Selon l'orientation de l'embryon, on distinguera des coupes longitudinales sagittales passant par son plan de symétrie, donc asymétriques (fig. 2), et des coupes longitudinales frontales perpendiculaires à ce plan et donc symétriques (fig. 4) (JUGUET, 1973).

La surrection de la feuille passe par les étapes suivantes (1) soubassement foliaire (2) initium foliaire marqué par un bombement (3) primordium foliaire caractérisé par un bourrelet plus ou moins allongé et un début d'accroissement latéral et (4) ébauche foliaire. On a vu que presque tous les auteurs qui ont étudié ce sujet utilisent uniquement le terme de primordium, fournissant ainsi des descriptions imprécises. Nous avons donc employé le terme de feuille pour tous les stades et mesuré sa longueur en prenant comme point zéro le sommet du méristème caulinaire; sa mesure est donc négative quand elle ne dépasse par le méristème, positive quand elle le dépasse.

Par ailleurs, mais cela a moins d'importance, la feuille est arbitrairement comptabilisée dans le nombre de feuilles total de l'embryon quand elle dépasse le méristème caulinaire.

Nous avons également compté le nombre de racines adventives séminales.

Les 5 hybrides suivants ont été étudiés :

- F7 $\times$ F2 : hybride simple précoce à grain corné constitué de 2 lignées issues de la population "Lacaune ». Ces lignées (ou l'une d'elles), utilisées dans la plupart des hybrides précoces cultivés en Europe, ont amélioré la tolérance aux températures basses du printemps.

- WH $\times$ WJ : hybride simple précoce à grain denté, d'origine américaine, assez sensible aux températures basses du printemps, constitue en croisement avec F7 × F2, l'hybride double INRA 200 .

- A632 × W117 : hybride simple demi-précoce, caractéristique des hybrides précoces du corn-belt américain, cultivé en France.

- W64A x F546 (INRA 508) : hybride simple demi-tardif typique du Sud-Ouest de la France. 
- B73 $\times$ Mo17 : hybride simple tardif, typique du corn-belt américain, trop tardif pour être cultivé pour le grain en France.

\section{RÉSULTATS}

Nous présentons dans la planche qui suit 4 dessins semi-schématiques d'embryons adultes.

Les figures 1 et 2 se rapportent à l'hybride W64A $\times$ F546. La coupe transversale (fig. 1) montre le coléoptile avec ses 2 faisceaux libéro-ligneux (fllc); celui-ci entoure le système foliaire formé de 4 feuilles plus ou moins développées et d'une $5^{e}$ encore à l'état de primordium. L'insertion distique des feuilles est bien visible; il existe un plan de symétrie vertical.

La figure 2 qui est une coupe longitudinale effectuée selon ce plan (plan sagittal) montre le primordium de F5 à gauche du méristème caulinaire ; ce dernier a été noirci et sa surface arbitrairement limitée vers le bas par une ligne horizontale.

Les 2 autres coupes (fig. 3 et 4) sont celles de l'hybride A632 $\times$ W117. On retrouve une disposition similaire sur la coupe transversale mais les 2 dernières feuilles (F4 et F5) sont considérablement plus développées tandis que F6 a atteint le stade de primordium. La figure 4 est une coupe longitudinale frontale; la zone centrale y apparaît donc comme symétrique et montre les 2 parties latérales du primordium de F6.

La simple comparaison de ces 2 séries de dessins montre à l'évidence que l'organogenèse foliaire de A632 $\times$ W117 est plus avancée que celle de W64A $\times$ F546; le $2^{\mathrm{e}}$ possède seulement 4 feuilles, le $1^{\mathrm{e}}, 5$, nettement plus développées.

Les résultats quantitatifs sont présentés dans les tableaux 1 et 2 par une série de mesures respective- ment pour F4 et F5 (et F6). La différence entre les effectifs mesurés pour F4, d'une part, et pour F5 et F6, d'autre part, est due aux observations qui ont été réalisées sur des coupes longitudinales pour la $1^{\text {re }}$, et transversales sur les autres. Le sommet du méristème caulinaire constitue le point zéro.

Nos observations ne permettent pas de différencier W64A $\times$ F546 et B73 $\times$ Mol7 qui montrent toujours 4 feuilles. F7 $\times$ F2 est plus développé et possède exceptionnellement 5 feuilles. L'organogenèse de $\mathrm{WH} \times \mathrm{WJ}$ et de A632 $\times$ W117 est encore plus avancée ; WH $\times$ WJ possède 5 feuilles dans un peu plus de la moitié des échantillons, tandis que F6 est régulièrement annoncée par des cloisonnements périclines à la base du méristème ou, exceptionnellement, par un léger bourrelet. Enfin, A632 $\times$ W117 montre le système foliaire le plus développé, la $5^{\mathrm{e}}$ feuille est toujours présente et la $6^{\mathrm{e}}$ représentée par un primordium plus ou moins développé sauf chez de rares échantillons (3 sur 22) où elle est indiscernable.

Le classement des 5 hybrides est confirmé par l'étude du système radiculaire adventif dans la graine mûre. Nous avons simplement compté le nombre de racines séminales adventives encore appelées secondaires par opposition à la racine primaire directement issue de la partie basale de l'embryon (JUGUET, 1971). Il est impossible de mesurer régulièrement l'état de leur développement car, tout en étant initiées latéralement, elles sont toujours obliques par rapport à l'axe embryonnaire ; seules quelques rares coupes longitudinales en montrent une dans son intégralité. Le tableau 3 résume nos résultats.

W64A $\times$ F546 et B73 $\times$ Mo17 qui possèdent un système foliaire relativement peu développé montrent donc au contraire un système radiculaire avancé tandis que l'équilibre inverse est réalisé chez les 3 autres hybrides malgré leurs relatives différences au niveau foliaire.

TABLEAU

Taille de la $4^{e}$ feuille (effectif observé). Size of the 4 th leaf (observed number).

\begin{tabular}{|c|c|c|c|c|c|}
\hline Taille en $\mu \mathrm{m}$ & W64A $\times$ F546 & $\mathrm{B} 73 \times \mathrm{Mo} 17$ & $\mathrm{~F} 7 \times \mathrm{F} 2$ & $\mathrm{WH} \times \mathrm{WJ}$ & $\mathrm{A} 632 \times \mathrm{W} 117$ \\
\hline $701-750$ & & & & & 1 \\
\hline $651-700$ & & & & & \\
\hline $601-650$ & & & & & 3 \\
\hline $551-600$ & & & & & 2 \\
\hline $501-550$ & & & & 1 & 4 \\
\hline $451-500$ & & & 1 & 3 & \\
\hline $401-450$ & & & 3 & 3 & 1 \\
\hline $351-400$ & & & 3 & & 2 \\
\hline $301-350$ & & 3 & 6 & 7 & \\
\hline $251-300$ & 4 & 6 & 3 & 2 & \\
\hline $201-250$ & 6 & 6 & 1 & & \\
\hline $151-200$ & 6 & 2 & & & \\
\hline $101-150$ & 2 & 1 & & & \\
\hline Effectif & 18 & 18 & 17 & 16 & 13 \\
\hline Moyenne & 208 & 247 & 346 & 378 & 540 \\
\hline Ecart-type & 48,5 & 54,8 & 66,2 & 80,5 & 102,8 \\
\hline
\end{tabular}



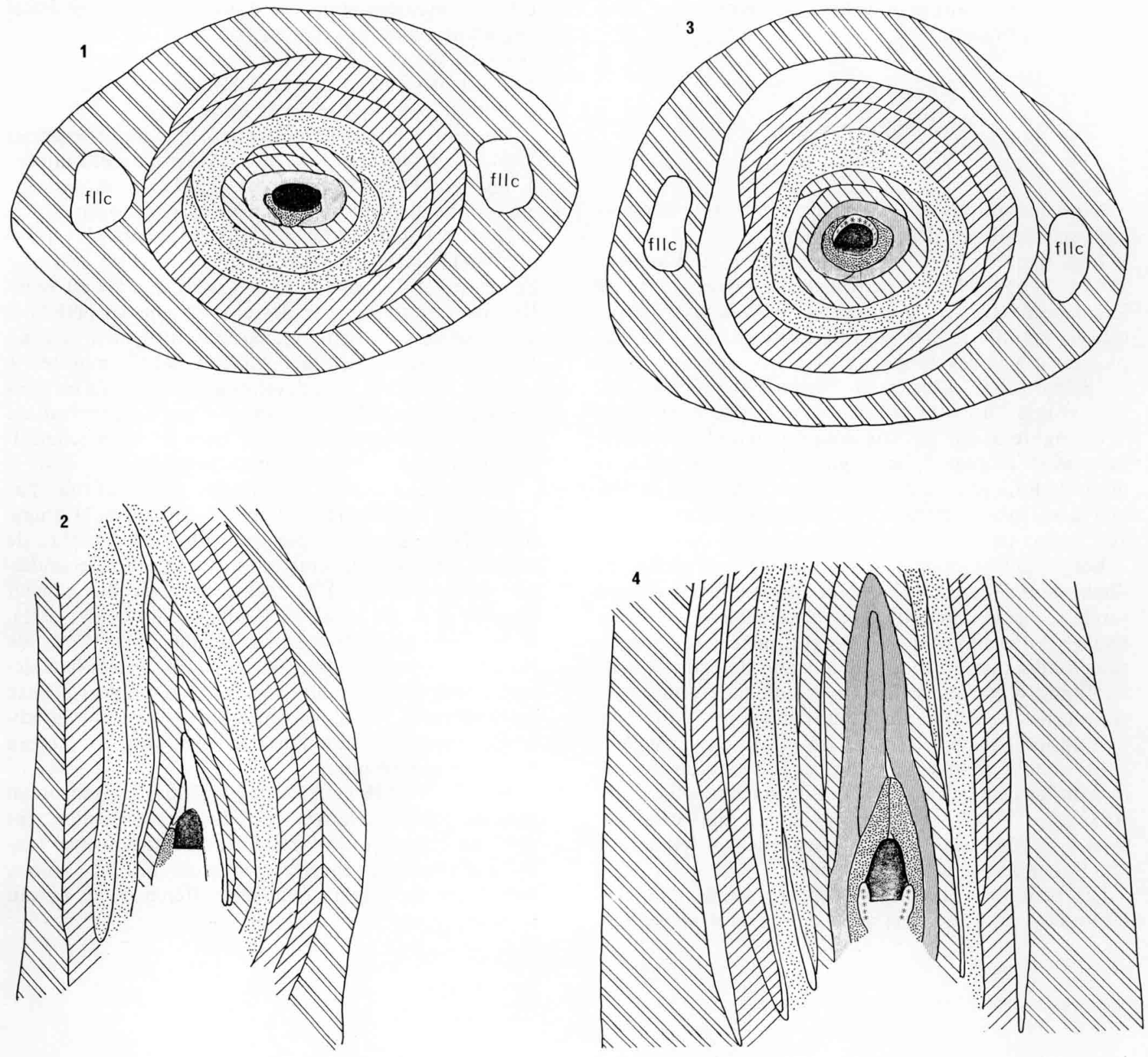

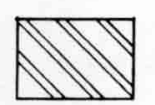

Coleoptile

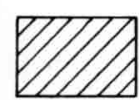

$F_{1}$ (1st leaf)
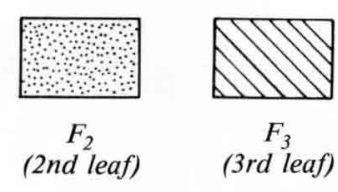

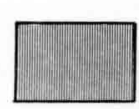

$F_{4}$

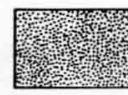

$F_{5}$ (4th leaf)

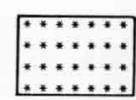

$F_{6}$ (6th leaf)

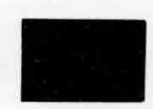

Méristème

Figure 3

W64A $\times$ F546: coupe transversale.

W64A $\times$ F546: cross section.

A632 $\times$ WII7 : coupe transversale.

A632 $\times$ W117 : cross section.

Figure 4

A632 $\times$ W117 : coupe longitudinale frontale. A632 $\times$ W117: Iongitudinal fronial section.

W64 $\times$ F546: coupe longitudinale (plan sagittal).

W64A $\times$ F546: longitudinal sagittal section 
TABLEAU 2

Taille de la $5^{e}$ feuille (et de la $\sigma^{e}$ feuille) par rapport au sommet du méristème (0) (effectif observé). Size of the 5 th leaf (and 6th leaf) in relation to meristem top (0) (observed number).

\begin{tabular}{|c|c|c|c|c|c|c|}
\hline \multicolumn{6}{|c|}{ F5 } & F6 \\
\hline Taille en $\mu \mathrm{m}$ & $\mathrm{W} 64 \mathrm{~A} \times \mathrm{F} 546$ & $\mathrm{~B} 73 \times \mathrm{Mo17}$ & $\mathrm{F} 7 \times \mathrm{F} 2$ & $\mathrm{WH} \times \mathrm{WJ}$ & $\mathrm{A} 632 \times \mathrm{W} 117$ & $\mathrm{~A} 632 \times \mathrm{W} 117$ \\
\hline+200 & & & & & 1 & \\
\hline+180 & & & & & & \\
\hline+160 & & & & & & \\
\hline+140 & & & & 1 & 4 & \\
\hline+120 & & & & & 3 & \\
\hline+100 & & & & & 7 & \\
\hline+80 & & & & 1 & 5 & \\
\hline+60 & & & & 4 & 3 & \\
\hline+40 & & & & 3 & 1 & \\
\hline$+\quad 20$ & & & 1 & 5 & & \\
\hline 0 & & & 2 & 3 & & \\
\hline-20 & & 2 & 6 & 2 & & \\
\hline-40 & 1 & 4 & 9 & & & \\
\hline-60 & 11 & 8 & 7 & 4 & & \\
\hline-80 & 9 & 9 & 3 & & & \\
\hline-100 & 3 & 1 & 2 & & & 1 \\
\hline-120 & & & & & & 6 \\
\hline-140 & & & & & & 11 \\
\hline-160 & & & & & & 1 \\
\hline Non discernable & & & & & & 3 \\
\hline Effectif & 24 & 24 & 30 & 23 & 24 & 22 \\
\hline Moyenne & $-71,6$ & $-62,5$ & -44 & $+17,4$ & +102 & -133 \\
\hline Ecart-type & 15,5 & 20,7 & 28,5 & 50,2 & 34,8 & 13,7 \\
\hline
\end{tabular}

TABLEAU 3

Nombre de racines séminales adventives (effectif observé).

Number of adventitions seed roots (observed number).

\begin{tabular}{cccccc}
\hline \hline Nombre de racines adventives & W64A $\times$ F546 & B73 $\times$ Mo17 & F7 $\times$ F2 & WH $\times$ WJ & A632 $\times$ W117 \\
\hline 2 & 0 & 0 & 17 & 0 & 25 \\
3 & 15 & 18 & 0 & 0 & 0 \\
4 & 6 & 11 & 0 & 0 \\
\hline
\end{tabular}

\section{DISCUSSION ET CONCLUSION}

Si l'on considère les travaux de BLOC et al. (1979), on peut donc estimer que le " capital embryonnaire" (pour reprendre l'expression de SASS, 1951) mis en réserve dans la graine est déterminant pour la nouvelle génération puisque l'on retrouve dans l'embryon adulte l'avantage observé sur la jeune plantule et sur la plante adulte de l'hybride $\mathrm{A} 632 \times \mathrm{W} 117$. Ce capital ne doit pas être évalué seulement en nombre de feuilles développées, mais doit également comptabiliser les premiers stades de l'organogenèse foliaire et surtout la dimension des organes. On pourrait parler de "surface foliaire présente dans l'embryon ».

Enfin la variabilité observée en ce qui concerne le nombre de racines séminales adventives est très intéressante, mais ce caractère paraît malheureusement lié négativement au nombre de feuilles. Cela semblerait reproduire sur l'embryon adulte l'équilibre parfois constaté aux premiers stades de la segmentation.
Cette étude doit être poursuivie dans plusieurs directions :

- observation d'un plus grand nombre de génotypes pour mieux apprécier la variabilité génétique et préciser la relation développement foliaire-développement racinaire ;

- étude de l'influence de la semence : " effet génétique » du parent femelle, mais aussi «qualité de la semence " : maturation et poids ;

- étude de la transmission de ce caractère par l'observation des lignées constituant ces hybrides ;

- étude de la correspondance entre les observations embryonnaires et le comportement des plantules et, si possible, des plantes adultes en conditions contrôlées et au champ. 


\section{RÉFÉRENCES BIBLIOGRAPHIQUES}

Abbe E. C., Stein O. L., 1954. The growth of the shoot apex in maize embryogeny. Am. J. Bot., 41, 285-296.

Bell M. E., 1953. The development of the embryo of Zea in relation to position on the ear. Iowa St. Coll. J. Sci., 29, 133-139.

Biscoe P. V., Gallagher J. N., 1977. Weather, dry-matter, production and yield, 75-100. In : Lansberg J. J. \& Cutting C. V. : "Environmental effects on crop physiology". Proc. Symp. Long Asthon, $388 \mathrm{p}$.

Bloc D., Bonhomme R., Gay J. P., Girardin P., Gouet J. P., 1979. Etude de l'influence de la température sur le développement du maîs. Résultats 1978-1979. Brochure AGPM, 34 p.

Bonhomme R., Ruget F., Derieux M., Vincourt P., 1982. Relations entre production de matière sèche aérienne et énergie interceptée chez différents génotypes de maïs. C. R. Acad. Sci., Paris, t. 294, série III, 393-398.

Bonnet O. T., 1953. Developmental morphology of the vegetative and floral shoot of maize. Bull. Univ. Ill. Agric. Exp. Stn., $\mathrm{n}^{\circ} 568$, $45 \mathrm{p}$.

Fairchild R. S., 1953. Comparative development of the embryo of inbred and hybrid maize. Iowa State Coll. J. Sci., 27, 381-405.

Gosse G., Varlet-Grancher C., Bonhomme R., Chartier M., Allirand J. M., Lemaire G., 1986. Production maximale de matière sèche et rayonnement solaire intercepté par un couvert végétal. Agronomie, 6 (1).

Juguet M., 1971. Embryogenèse des Cyperaceae et des familles voisines. Application à la connaissance de l'embryon des Monocotylédones et à la systématique. Thèse d'Etat, 5784, Univ. Picardie, $300 \mathrm{p}$.

Juguet M., 1973. Expression précoce de la monocotylédonie et mise en place du cotylédon et du point végétatif de la tige chez quelques Monocotylédones, avec quelques remarques sur les types de symétrie des embryons de Monocotylédones et de Dicotylédones. Bull. Soc. Bot. Fr., Mémoires, 337-354.

Kiesselbach T. A., 1949. The structure and reproduction of corn. Nebr. Agric. Exp. Stn. Res. Bull., 161, 1-96.

Lomasson R. C., 1977. Some morphological aspects of the corn stem. Proc. Nebr. Acad. Sci. affil. Soc., 87, 17.

Martin J. N., Hershey A. L., 1935. The ontogeny of the maize plant. The early differentiation of stem and root structures and their morphological relationships. Iowa State Coll. J. Sci., 9, 489-503.

Monteith J. L., 1977. Climate and the efficiency of crop production in Britain. Phil. Trans. R. Soc. London, B, 281, 277-294.

Randolph L. F., 1936. Developmental morphology of the caryopsis in maize. J. Agric. Res., 53, 881-916.

Sass J. E., 1951. Comparative leaf number in the embryos of some type of maize. Iowa State Coll. J. Sci., 25, 509-512.

Sass J. E., 1976. Development of the caryopsis, 89-110. In : G. F. Sprague: "Corn and Corn Improvement", Am. Soc. Agron., $763 \mathrm{p}$.

Seung Keun Jong, Brewbaker J. L., Chong Hee Lee, 1982. Effects of solar radiation on the performance of maize in 41 successive monthly plantings in Hawaii. Crop Sci., 22, 13-18.

Shafer J., 1944. The relation of embryo axis weight to heterosis. Am. J. Bot., 31, 503-506.

Varlet-Grancher C., 1982. Analyse du rendement de la conversion de l'énergie solaire par un couvert végétal. Thèse $\mathrm{n}^{\circ} 2593$, Univ. Paris-Sud, Orsay, 144 p. 